\title{
Decreased vascular lesion formation in mice with inducible endothelial-specific expression of protein kinase Akt
}

\author{
Yasushi Mukai, ${ }^{1}$ Yoshiyuki Rikitake, ${ }^{1}$ Ichiro Shiojima, ${ }^{2}$ Sebastian Wolfrum, ${ }^{1}$ \\ Minoru Satoh, ${ }^{1}$ Kyosuke Takeshita, ${ }^{1}$ Yukio Hiroi, ${ }^{1}$ Salvatore Salomone, ${ }^{3}$ \\ Hyung-Hwan Kim, ${ }^{1}$ Laura E. Benjamin, ${ }^{4}$ Kenneth Walsh, ${ }^{2}$ and James K. Liao ${ }^{1}$
}

\begin{abstract}
${ }^{1}$ Vascular Medicine Research Unit, Brigham and Women's Hospital, Harvard Medical School, Boston, Massachusetts, USA. 'Molecular Cardiology Unit, Whitaker Cardiovascular Institute, Boston University School of Medicine, Boston, Massachusetts, USA. ${ }^{3}$ Stroke and Neurovascular Regulation Laboratory, Massachusetts General Hospital, and 4Department of Pathology, Beth Israel Deaconess Medical Center, Harvard Medical School, Boston, Massachusetts, USA.
\end{abstract}

To determine whether endothelial Akt could affect vascular lesion formation, mutant mice with a constitutively active Akt transgene, which could be inducibly targeted to the vascular endothelium using the tet-off system (EC-Akt Tg mice), were generated. After withdrawal of doxycycline, EC-Akt Tg mice demonstrated increased endothelial-specific Akt activity and NO production. After blood flow cessation caused by carotid artery ligation, neointimal formation was attenuated in induced EC-Akt Tg mice compared with noninduced EC-Akt Tg mice and control littermates. To determine the role of eNOS in mediating these effects, mice were treated with $N^{\omega}$-nitro-L-arginine methyl ester (L-NAME). Neointimal formation was attenuated to a lesser extent in induced EC-Akt Tg mice treated with L-NAME, suggesting that some of the vascular protective effects were NO independent. Indeed, endothelial activation of Akt resulted in less EC apoptosis in ligated arteries. Immunostaining demonstrated decreased inflammatory and proliferative changes in induced EC-Akt Tg mice after vascular injury. These findings indicate that endothelial activation of Akt suppresses lesion formation via increased NO production, preservation of functional endothelial layer, and suppression of inflammatory and proliferative changes in the vascular wall. These results suggest that enhancing endothelial Akt activity alone could have therapeutic benefits after vascular injury.

\section{Introduction}

The endothelium plays an important role in regulating vascular integrity. Under normal conditions, the vascular endothelium secretes a variety of vasoactive substances including $\mathrm{NO}$ and prostacyclin, which protect the vascular wall against vasoconstriction, inflammatory and proliferative changes, and thrombus formation $(1,2)$. Recent studies suggest that impairment of endothelial function, as observed in the presence of cardiovascular risk factors, is not only a marker but also contributes to the pathogenesis of cardiovascular disease. Thus, improving endothelial function is an important therapeutic target for reducing vascular diseases such as arteriosclerosis and atherosclerosis $(3,4)$.

Numerous signaling pathways in ECs, such as those involving $\mathrm{G}$ protein-coupled receptors, MAPKs, protein kinase B/Akt, and small G proteins, contribute to endothelial function. In particular, intracellular signaling pathways mediated by protein kinase Akt are involved in the regulation of cell survival, proliferation, migration, glucose metabolism, and gene expression $(5,6)$. In ECs and possibly endothelial progenitor cells (EPCs), a variety of

Nonstandard abbreviations used: EC-Akt Tg mouse, endothelial-specific inducible myristoylated Akt1 transgenic mouse; EEL, external elastic lamina; EPC, endothelial progenitor cell; FKHR, Forkhead in rhabdomyosarcoma; GSK-3, glycogen synthase kinase-3; IEL, internal elastic lamina; L-NAME, $N^{\omega}$-nitro-L-arginine methyl ester; MLEC, mouse lung EC; myrAkt, myristoylated Akt1; PCNA, proliferating cell nuclear antigen; PECAM-1, platelet endothelial cell adhesion molecule-1; tTA, tetracyclinecontrolled transcriptional activator; VE-cadherin, vascular endothelial cadherin. Conflict of interest: The authors have declared that no conflict of interest exists. Citation for this article: J. Clin. Invest. 116:334-343 (2006). doi:10.1172/JCI26223. growth factors, such as VEGF and insulin; HMG-CoA reductase inhibitors, or statins; and fluid shear stress activate the Akt pathway in a PI3K-dependent manner (6). The activation of Akt leads to the phosphorylation and activation of eNOS and increased production of $\mathrm{NO}(6-8)$. Other downstream targets of Akt, such as glycogen synthase kinase-3 (GSK-3) (9), the Forkhead in rhabdomyosarcoma (FKHR) family of transcriptional factors (10), and BAD (11), regulate cellular metabolism and survival of ECs in response to growth factors. Thus, it is likely that the endothelial Akt pathway is critically involved in many biological aspects of the vascular wall (6). Indeed, Akt signaling plays an important role in the proliferation and migration of ECs, both of which may contribute to some of its proangiogenic effects (6, 12-14). However, it is not known whether activation of Akt in the endothelium alone could alter the process of lesion formation in the vascular wall after injury.

Given that Akt is important in endothelial function and survival, we hypothesized that endothelial-specific activation of Akt signaling may be protective against pathological processes associated with endothelial dysfunction. To test this hypothesis, we generated $\mathrm{Tg}$ mice that express a constitutively active mutant form of Akt, which can be targeted specifically and inducibly to ECs (EC-Akt Tg mice) (15). This was accomplished using the vascular endothelial cadherin (VE-cadherin) promoter (VE-cadherin-tTA) coupled to the tetracycline-controlled gene expression (tet-myrAkt) system (tet-off system) $(16,17)$. Here we show that in EC-Akt Tg mice, the increase in Akt activity in the endothelium alone protects the vascular wall from inflammatory and proliferative changes following vascular injury. 
A

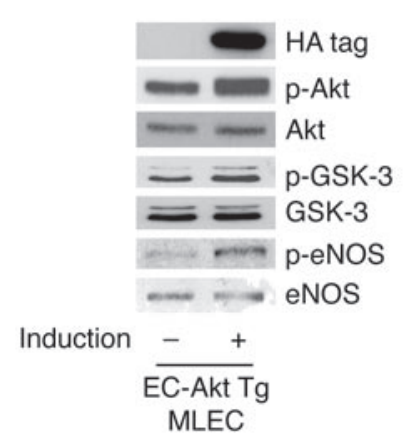

B
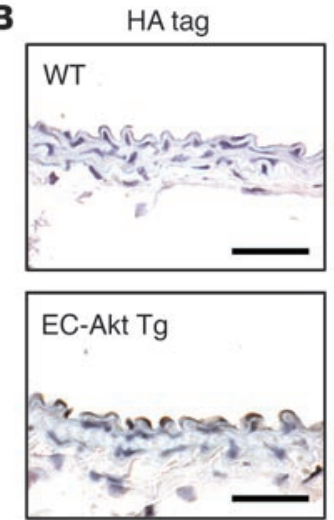

C

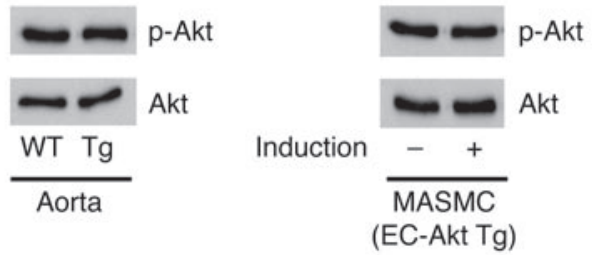

Figure 1

Endothelial-specific expression of inducible myrAkt transgene in EC-Akt Tg mice. (A) Western blots in primary MLEC cultures. The expression of myrAkt transgene was determined by the expression of HA tag. ECs from EC-Akt Tg mice were divided into 2 groups, with and without myrAkt gene induction. Phosphorylated forms of Akt, GSK-3, or eNOS were determined in these ECs. Each blot is representative of 3 independent experiments. (B) Endothelial expression of myrAkt in vivo. Immunostaining in carotid artery, showing HA-tag expression in the endothelial layer of an induced EC-Akt Tg mouse. Scale bar: $50 \mu \mathrm{m}$. (C) Immunoblots showing phosphorylation of Akt in aortae from induced EC-Akt Tg mice (left) and in primary cultures of aortic SMCs isolated from induced EC-Akt Tg mice (MASMC, right).

\section{Results}

Endothelial-specific expression of constitutively active Akt in EC-Akt $\mathrm{Tg}$ mice. The expression of constitutively active, myristoylated Akt1 (myrAkt) was confirmed by detection of the HA tag in primary EC cultures isolated from EC-Akt Tg mice (Figure 1A). For gene induction, doxycycline was removed from the culture medium for 2 days. The phosphorylation of both Akt and its major substrates GSK-3 and eNOS was increased in ECs with gene induction (Figure 1A). Endothelial expression of the HA tag could also be detected in vivo by immunostaining of the carotid artery from EC-Akt Tg mice after gene induction (Figure 1B). As a control for endothelial specificity, phosphorylation of Akt was not different in the aortae of WT and EC-Akt Tg mice and was not increased following gene induction in aortic SMCs isolated from EC-Akt Tg mice (Figure 1C). For in vivo gene induction, withdrawal of doxycycline for 3 days from the drinking water was sufficient. Compared with WT mice given a similar protocol with doxycycline, the mean systolic blood pressure $(\mathrm{mmHg})$ in EC-Akt Tg mice as measured by tail-cuff method 4 weeks after gene induction was not different $(126.3 \pm 3 \mathrm{mmHg}$ in WT mice and $125.8 \pm 3 \mathrm{mmHg}$ in EC-Akt Tg mice, $n=10$ in each group, $P=0.882$ ). The systolic blood pressure of noninduced EC-Akt $\mathrm{Tg}$ mice (i.e., no doxycycline withdrawal) was similar to that of WT mice.

Endothelial NO production in EC-Akt Tg mice. To determine the levels of endothelial NO production in EC-Akt Tg mice, we measured $\mathrm{NO}_{2}$ accumulation in the conditioned media in primary EC cultures isolated from EC-Akt $\mathrm{Tg}$ mice. $\mathrm{NO}_{2}$ accumulation was significantly enhanced in ECs from EC-Akt Tg mice with gene induction compared with those without gene induction (Figure 2A). To further determine NO bioactivity in a tissue level, we performed isometric tension recordings in aorta isolated from EC-Akt Tg mice after gene induction. Endothelial-dependent contractions in response to a NOS inhibitor, $N^{\omega}$-nitro-L-arginine methyl ester (L-NAME), which indicates the basal level of endothelial NO release, were higher in induced EC-Akt Tg mice compared with noninduced or WT mice. These findings indicate that endothelialspecific activation of Akt leads to increased production and bioactivity of NO in vascular tissues.
Endothelial-specific Akt activation attenuates neointimal formation following injury. To investigate the effect of endothelial-specific activation of Akt on vascular lesion formation, we used a mouse carotid artery ligation model as a model for non-endotheliumdenuding neointimal formation (18). The endothelium has been shown to play a significant role in the development of vascular lesions in this model $(18,19)$. Furthermore, lesion formation in this model is dependent on alteration of fluid shear stress (18), which is a major physiological activator of endothelial Akt signaling and eNOS $(20,21)$.

Neointimal formation with an accompanying decrease in luminal area was observed in WT mice, single-Tg mice (i.e., tet-myrAkt), and noninduced EC-Akt Tg mice at 4 weeks after carotid artery ligation (Figures $3 \mathrm{~A}$ and 4 ). The extent of neointimal formation
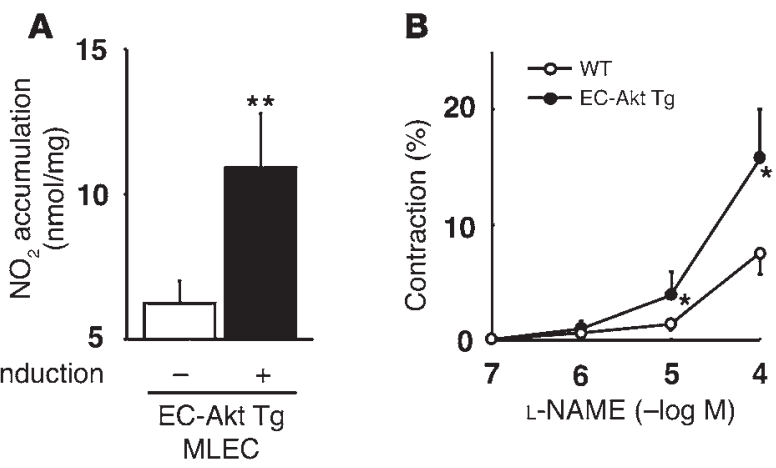

\section{Figure 2}

Endothelial NO production and bioactivity in EC-Akt Tg mice. (A) NO production in MLECs from induced EC-Akt Tg mice. Nitrite $\left(\mathrm{NO}_{2}\right)$ was measured in ECs under serum-starved conditions with or without myrAkt transgene induction. Results are expressed as mean \pm SEM. $n=8$ in each group. ${ }^{* *} P<0.01$ versus cells without gene induction. (B) Force measurement in isolated mouse aorta. Endothelial-dependent contractions in response to L-NAME were determined. Results are expressed as mean \pm SEM. $n=6$ in each group. ${ }^{*} P<0.05$ versus WT. 
A

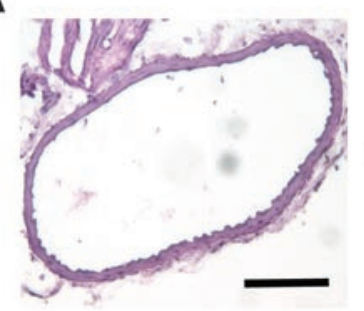

WT (sham)

WT

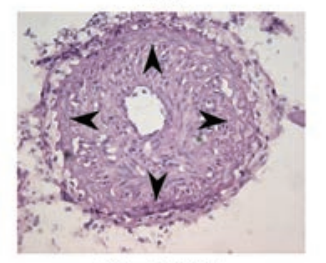

EC-Akt Tg

induction (-)

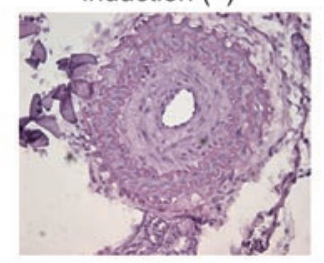

WT

B + L-NAME

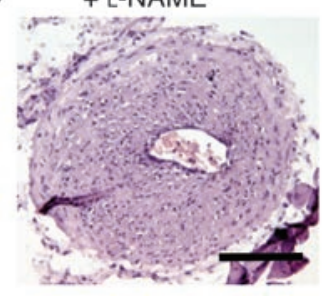

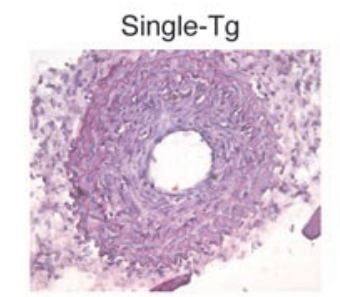

EC-Akt Tg

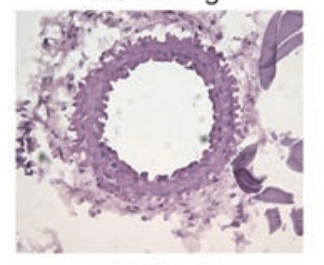

EC-Akt Tg + L-NAME

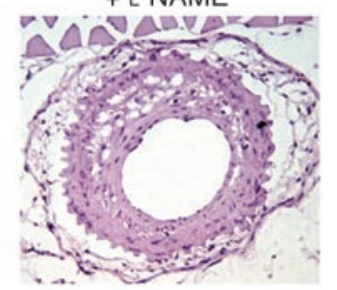

was comparable among these 3 control groups. The predominant cellular component of the neointima was VSMCs, as determined by robust positive staining for $\alpha$-SMA (data not shown). In contrast, in induced EC-Akt Tg mice, neointima formation as determined by intimal area and intima/media ratio was inhibited (Figures $3 \mathrm{~A}$ and 4 ). This corresponded with improvement in luminal area of induced EC-Akt $\mathrm{Tg}$ mice compared with control mice. However, the media and total vascular areas were not different between the animal groups after carotid artery ligation. These results indicate that endothelialspecific activation of Akt attenuates lesion formation following blood flow cessation.

Role of NO in vascular lesion formation. To determine the potential role of endothelium-derived NO in mediating the decreased vascular lesion formation observed in EC-Akt Tg mice, we performed carotid ligation in EC-Akt Tg mice treated with the NOS inhibitor L-NAME $(1 \mathrm{mg} / \mathrm{ml}$ in drinking water). Treatment with L-NAME increased blood pressure to a similar extent in WT and induced EC-Akt Tg mice (data not shown). Although treatment with L-NAME also increased

\section{Figure 3}

Photomicrographs of ligated mouse carotid arteries. (A) Photomicrographs of carotid arteries 4 weeks after ligation (H\&E staining) in WT, single-Tg (tet-myrAkt), noninduced EC-Akt Tg, and induced EC-Akt Tg mice. Arrowheads indicate internal elastic lamina. (B) Photomicrographs of H\&E staining of carotid arteries 4 weeks after ligation in WT and induced EC-Akt Tg mice treated with L-NAME. Scale bars: $100 \mu \mathrm{m}$.

neointima formation in both WT and induced EC-Akt Tg mice, the extent of neointima formation with L-NAME treatment was considerably less in induced EC-Akt $\mathrm{Tg}$ mice compared with that in WT mice (Figures 3B and 4). These results suggest that endothelium-derived NO contributes to some, but not all, of the decreased vascular lesion formation observed in induced EC-Akt Tg mice.

Prevention of EC apoptosis in EC-Akt Tg mice. Physiological shear stress promotes EC survival through activation of Akt (20). In our carotid ligation model, it is likely that the cessation of blood flow leads to a decrease in Akt activity and EC survival. To determine whether the activation or preservation of Akt signaling could promote EC survival despite decreases in laminar shear stress, we measured the expression of cleaved (activated) caspase-3, which is regulated by Akt and is an indicator of the apoptotic process, in noninduced and induced EC-Akt Tg mice $(22,23)$. In mouse lung ECs (MLECs) isolated from these EC-Akt Tg mice, apoptosis was induced by serum starvation as exhibited by the increased expression of cleaved caspase-3 (Figure 5A). Many cells with cleaved caspase-3 expression showed shrinkage of nuclei, a typical change seen in apoptotic cells. In contrast to MLECs from noninduced EC-Akt Tg mice, the expression of cleaved caspase- 3 after serum starvation was substantially attenuated in MLECs derived from induced EC-Akt Tg mice (Figure 5A). Similar findings were also seen in ECs isolated from aortae of EC-Akt $\mathrm{Tg}$ mice (Figure $5 \mathrm{~B}$ ), suggesting that increased Akt activity by the transgene confers similar survival benefits to ECs from different vascular beds. These findings correlated with Western blotting analysis showing decreased expression of cleaved caspase- 3 in ECs from induced EC-Akt Tg mice (Figure 5A). These results indicate that the activation of Akt signaling in induced EC-Akt Tg mice is sufficient to prevent apoptotic cell death of ECs.
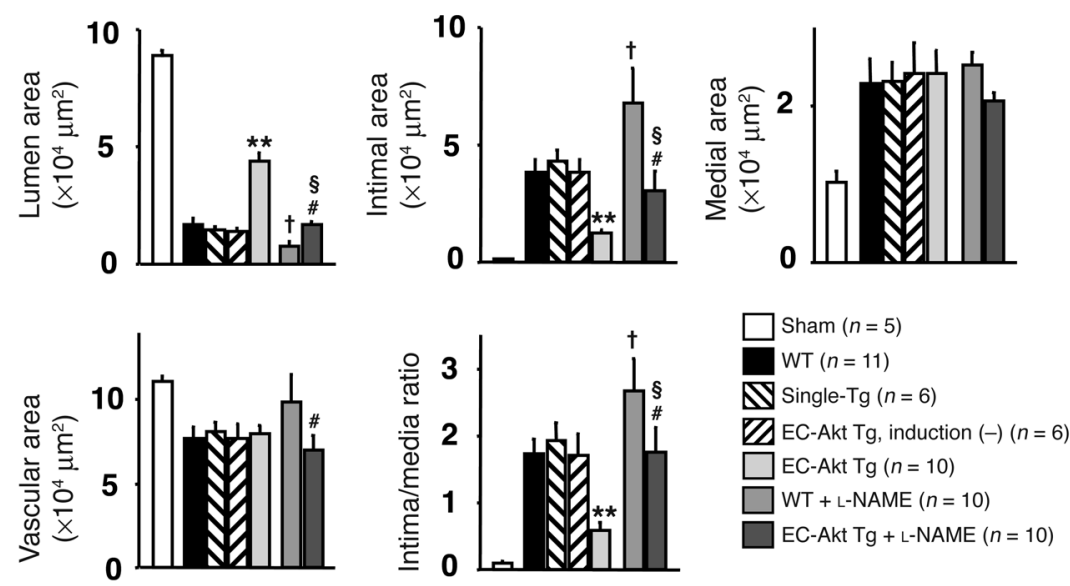

Figure 4

Quantitative analysis of vascular lesions in ligated carotid arteries. Results are expressed as mean \pm SEM. Number of animals in each group is as indicated. ${ }^{* \star} P<0.01$ versus WT, single-Tg, and noninduced EC-Akt Tg. ${ }^{\dagger} P<0.05$ versus WT, single-Tg, and noninduced EC-Akt Tg. ${ }^{\#} P<0.05$ versus WT + L-NAME. $\$ P<0.05$ versus EC-Akt Tg. 
A

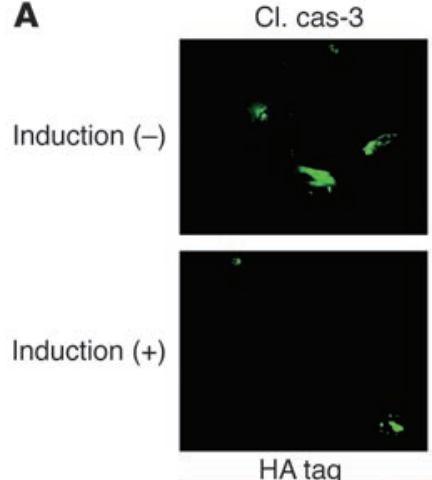

HA tag

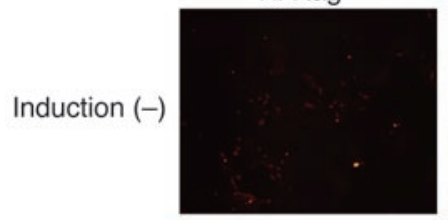

Induction (+)

B

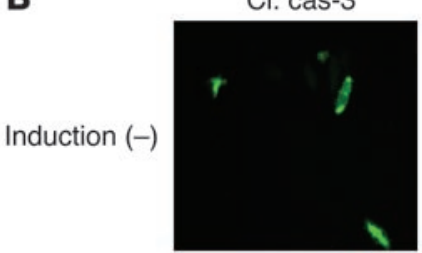

Cl. cas-3

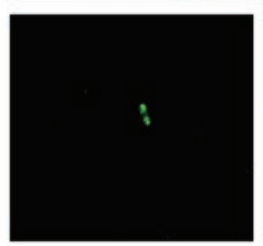

Hoechst
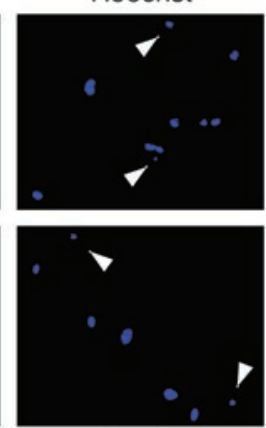

Hoechst
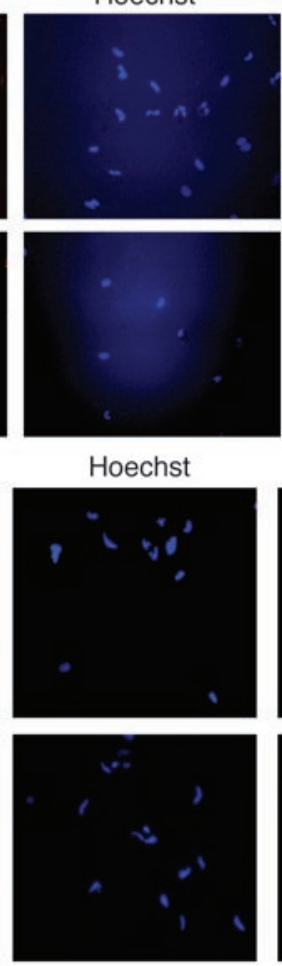

Merge
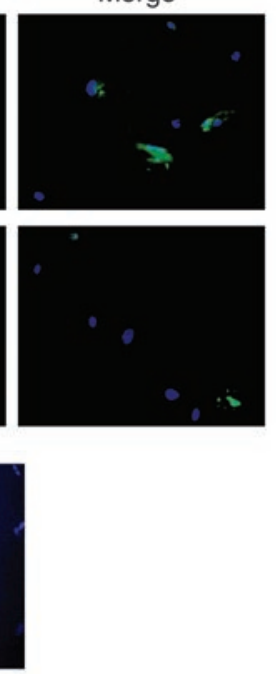

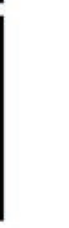
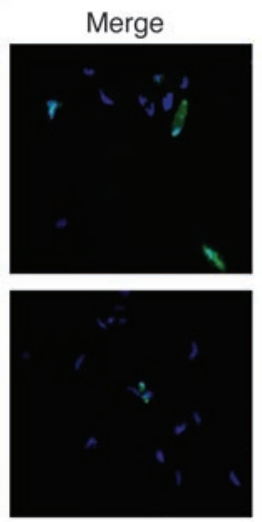
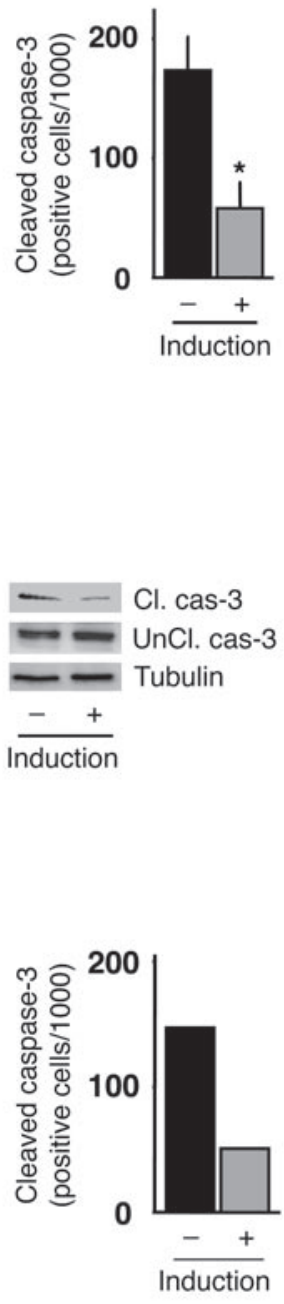

\section{Figure 5}

EC apoptosis. (A) Immunocytochemistry and immunoblotting for cleaved (activated) caspase-3. MLECs from induced and noninduced EC-Akt Tg mice were examined (upper panels). To induce apoptosis, a serum deprivation method for 24 hours was used. Positive staining for cleaved caspase-3 (green) is noted in cells without gene induction. Results are expressed as mean \pm SEM. $n=3$ in each group. ${ }^{*} P<0.05$ versus ECs from EC-Akt Tg mice without induction. Counterstaining with Hoechst 33258 shows shrinkage of nuclei in some cells with cleaved caspase3 expression (arrowheads). Expression of myrAkt was confirmed by detection of the HA tag (red) (lower left panel). Representative Western blots for cleaved and noncleaved caspase-3 in ECs with or without myrAkt gene induction are shown in the lower right panel. (B) Immunocytochemistry for cleaved (activated) caspase3 in ECs isolated from aortae of induced and noninduced EC-Akt mice. A representative result from 3 separate experiments is shown. Cl. cas-3, cleaved caspase-3; UnCl. cas-3, uncleaved caspase-3. Magnification, $\times 400$.
Immunostaining of ligated carotid arteries showed that the expression of cleaved caspase- 3 was substantially less in induced EC-Akt Tg mice compared with WT mice (Figure 6, A and B). Using TUNEL staining as another indicator of apoptotic cells (22), we found fewer TUNEL-positive ECs in induced EC-Akt Tg mice compared with WT mice (Figure 7, A and B). Staining for platelet endothelial cell adhesion molecule-1 (PECAM-1/CD31), a constitutively expressed EC adhesion molecule (24), in the same sections used for cleaved caspase- 3 or TUNEL staining (double staining) showed an irregular endothelial layer in WT mice after ligation, suggesting loss of ECs. In contrast, the integrity of the endothelial layer appeared to be preserved in induced EC-Akt Tg mice (Figures 6 and 7). These findings indicate that endothelial-specific activation of Akt promotes EC survival leading to preservation of intact endothelial layer after blood flow cessation.

Functional analysis of endothelial layer. The functional integrity of the endothelial layer was examined in ligated carotid arteries. Evans blue dye permeates into the vascular wall of conduit arteries when the endothelium is injured or denuded $(25,26)$. Thus, incorporation of Evans blue dye in the vessel could be a useful marker of endothelial integrity. However, the degree of Evans blue staining of the vessel wall may also be dependent on the level of blood flow, endothelial permeability, and vascular inflammation and, therefore, may not correlate entirely with endothelial integrity. Nevertheless, a week after carotid artery ligation, incorporation of Evans blue dye was observed in the vascular wall in WT mice, suggesting the presence of dysfunctional or detached endothelium. In contrast, incorporation of Evans blue dye was reduced in induced EC-Akt Tg mice (Figure 8). These findings suggest that the endothelial monolayer in induced EC-Akt Tg mice may be functionally more intact compared with that in WT mice after injury.

Inflammatory and proliferative changes in the vascular wall. An important initial step in vascular lesion formation is leukocyte recruitment to the vascular wall $(27,28)$. One week after carotid artery ligation, leukocyte accumulation was observed using an antibody against CD45 antigen. CD45-positive cells were detected in the vascular wall of WT mice and, to a much lesser extent, in the vascular wall of induced EC-Akt Tg mice (Figure 9, A and B). Using neutrophiland macrophage-specific antibodies, most of these leukocytes were found to be neutrophils, with some macrophages (data not shown). 
A

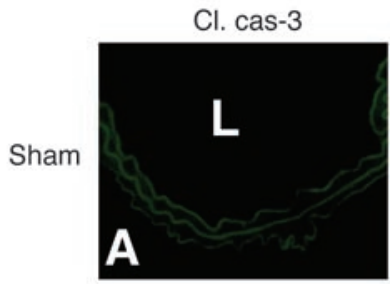

NT
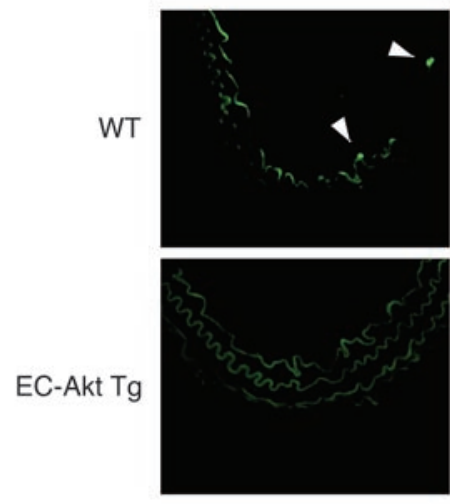

PECAM-1
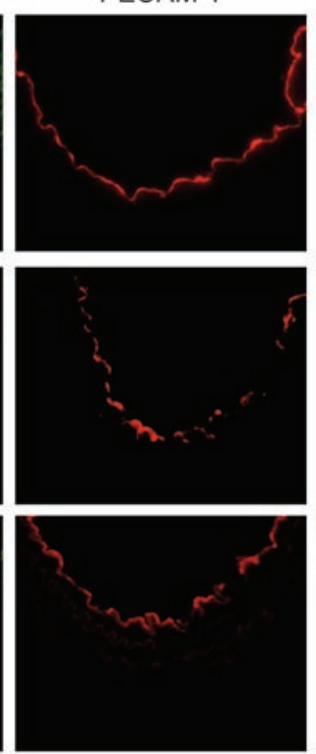
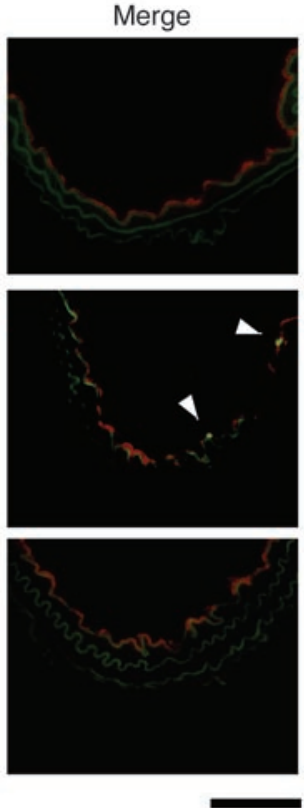

B

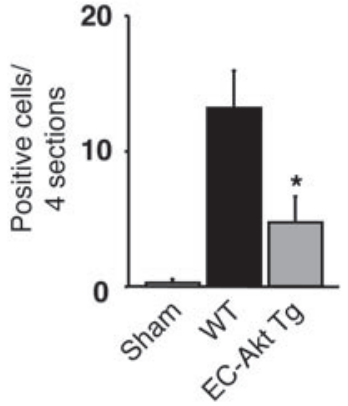

\section{Figure 6}

Immunostaining for cleaved (activated) caspase-3 in ligated carotid arteries. (A) Double-immunofluorescent staining in ligated carotid arteries showing ECs positively stained for cleaved caspase-3 (C. cas-3; green). Staining for PECAM-1 (red) was used to detect and assess the integrity of the endothelial layer. Scale bar: $50 \mu \mathrm{m}$. Arrowheads indicate positively stained ECs. L, lumen; A, adventitia. (B) The number of positive-staining cells in 4 separate sections per animal is quantified. Results are expressed as mean \pm SEM. $n=4$ in each group. ${ }^{*} P<0.05$ versus WT.

Similarly, the expression of ICAM-1 and VCAM-1 was increased in the endothelial layer in WT but not induced EC-Akt Tg mice after ligation (Figure 9A). These results indicate that endothelial-specific activation of Akt suppresses the expression of adhesion molecules and attenuates subsequent inflammatory changes in the vascular wall. These changes correlated with increased cellular proliferation in the vascular wall of WT mice and, to a lesser extent, in the vascular wall of induced EC-Akt Tg mice as determined by proliferating cell nuclear antigen (PCNA) staining (Figure 9, A and B).

\section{Discussion}

We have shown that inducible endothelial-specific activation of Akt signaling attenuates lesion formation and neointimal hyperplasia following non-endothelium-denuding vascular injury. These effects of Akt were associated with increased NO production, preservation of the endothelial layer, and subsequent suppression of inflammatory and proliferative changes in the vascular wall. These findings support our hypothesis that enhancement of endothelial Akt signaling alone protects the entire vascular wall under pathological conditions.

Recent studies suggest that the impairment of endothelial function is an important initial step in the pathogenesis of cardiovascular diseases. Consequently, the vascular endothelium has been regarded as a potential therapeutic target for pathological processes in the vascular wall $(1-4,27,28)$. Indeed, current therapeutic strategies such as statins and angiotensinconverting enzyme inhibitors may be beneficial, because they also improve endothelial function $(29,30)$. However, it is not known whether the modulation of endothelial function alone can influence lesion formation following vascular injury $(4,19$, 31). For example, increased Akt activity in VSMCs promotes vas- cular hypertrophy following balloon injury (32). In this regard, our results are somewhat unexpected in that modulation of endothelial function alone through enhancement of endothelial Akt signaling could have such a dramatic suppressive effect on vascular lesion formation.

VEGF, which acts as an EC-specific mitogen, has potent vascular-protective effects in vivo $(33,34)$. The beneficial effects of VEGF, in part, may be mediated by endothelial Akt signaling, since VEGF is a potent activator of this pathway (6). Statins are also able to activate the Akt pathway in ECs and EPCs (12, $35)$. It is possible, therefore, that some of the beneficial cholesterol-independent or pleiotropic effects of statins, such as their angiogenesis-promoting effects, could be due to the activation of Akt in ECs and EPCs. Other extracellular stimuli that activate endothelial Akt signaling, such as angiopoietin $(6,36)$ and adrenomedullin $(37,38)$, have been shown to be vascular protective, although the significance of endothelial Akt in their biological actions remains to be determined.

Akt phosphorylates and activates eNOS, resulting in increased endothelial NO production independent from the conventional calcium/calmodulin-dependent activation of eNOS $(7,8)$. We found that endothelial NO production is increased in primary EC cultures as well as in aortic segments isolated from EC-Akt Tg mice. These results are consistent with previous in vitro studies using the vector-mediated overexpression of constitutively active Akt $(7,8,39)$. Interestingly, in our model of neointimal hyperplasia, chronic inhibition of NOS with L-NAME exacerbated the proliferative response in EC-Akt Tg mice, albeit to a lesser extent compared with that in WT mice, indicating that NO-dependent and -independent endothelial mechanisms play an important role in the vascular-protective effects observed in EC-Akt Tg mice. 
A
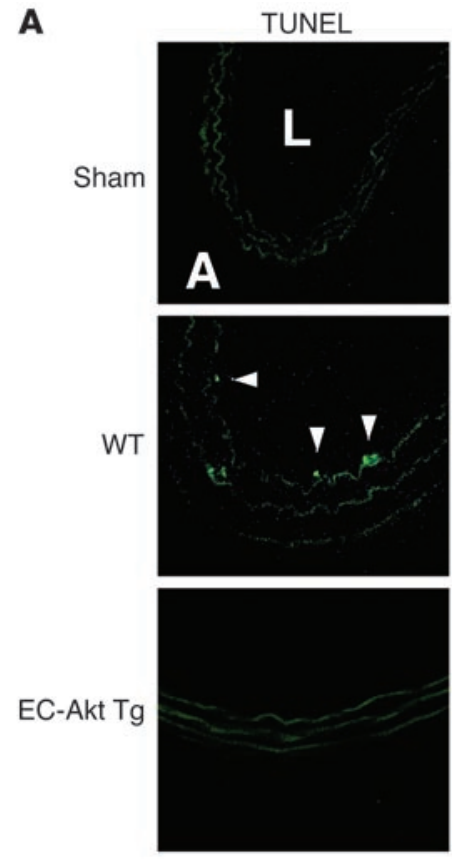

PECAM-1
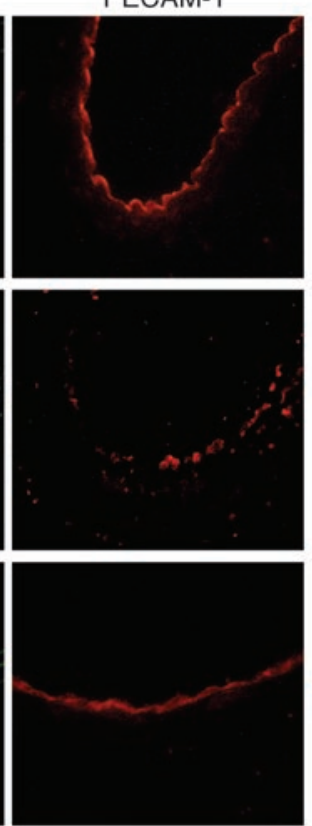
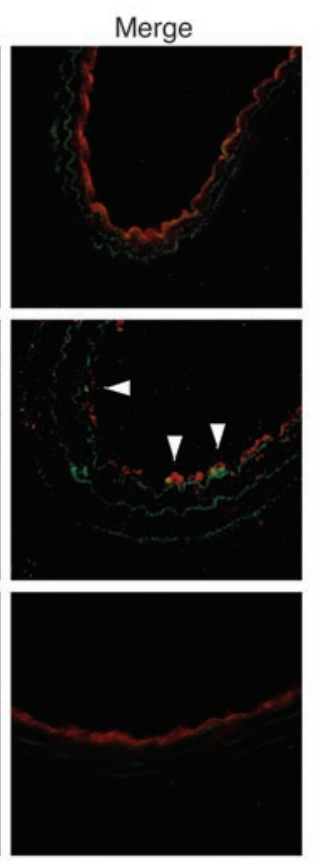

B

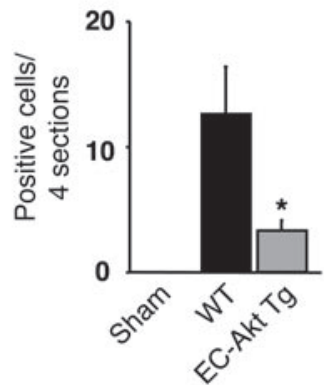

\section{Figure 7}

TUNEL staining in ligated carotid arteries. (A) Double-immunofluorescent staining to detect TUNEL-positive, apoptotic ECs in ligated arteries (green). Staining for PECAM-1 (red) was used to detect and assess the integrity of the endothelial layer. Scale bar: $50 \mu \mathrm{m}$. Arrowheads indicate apoptotic ECs. (B) The number of positive-staining cells in 4 separate sections per animal is quantified. Results are expressed as mean \pm SEM. $n=4$ in each group. ${ }^{*} P<0.05$ versus WT.

Akt functions as an important survival kinase in various cell types $(5,6)$, and previous studies have shown that physiological shear stress promotes EC survival through Akt signaling in vitro (20). Furthermore, lesion development in the vascular wall is associated with increased EC apoptosis and turnover that is induced by locally disturbed blood flow $(40,41)$. Thus, the findings from these studies suggest that vascular lesion formation may be more prominent in areas of decreased Akt signaling and increased EC apoptosis due to alteration of blood flow. Indeed, we found greater EC apoptosis in the ligated arteries from control mice compared with those from induced EC-Akt Tg mice, suggesting that preserved endothelial layer in these mice may protect against vascular lesion development.

To further examine the function of the endothelial layer, we applied Evans blue dye, which permeates through damaged endothelial layer $(25,26)$. Although incorporation of Evans blue dye in the vessel wall could be used to approximate decreased endothelial integrity, other factors such as regional blood flow, endothelial permeability, and inflammatory processes could alter the degree of Evans blue staining. Nevertheless, we observed reduced incorporation of the dye in the ligated vessels of induced EC-Akt Tg mice. These findings correlated with the degree of apoptosis and immunostaining for PECAM-1 and suggest that endothelial integrity and/or barrier function is preserved in induced EC-Akt Tg mice, most likely through improved EC survival and possibly re-endothelialization. Despite re-endothelialization following vascular injury, regenerated ECs appear somewhat dysfunctional (42-44). Thus, inhibition of EC apoptosis would be more favorable to preserving endothelial function than re-endo- thelialization. EC survival is also regulated by downstream targets of Akt, such as GSK-3, BAD, and the FKHR family of transcriptional factors $(6,9-11)$, and NO derived from eNOS (45). Thus, all of these downstream targets of Akt in addition to eNOS were probably involved in the preservation of the endothelial layer and subsequent vascular protection in EC-Akt Tg mice. Indeed, the $\mathrm{NO}$-independent mechanisms were evident in studies with mice treated with L-NAME. Taken together, these results indicate that a preserved and more functionally intact endothelial layer is associated with decreased vascular lesion formation.

An intact endothelium has antiinflammatory and antiproliferative effects on the vascular wall, which are important for vascular homeostasis. The development of vascular lesions is preceded by inflammatory and proliferative changes that are associated with endothelial dysfunction $(1,2,27,28)$. We found that the expression of endothelial adhesion molecules and the subsequent accumulation of leukocytes in the vascular wall were attenuated in induced EC-Akt Tg mice. These results are consistent with a previous study showing that activation of Akt suppresses adhesion molecule expression in vitro (46). The increased production of NO by the Akt induction probably plays an important role in the suppression of inflammatory changes, since NO negatively regulates the expression of adhesion molecules $(27,47)$. The Akt-mediated preservation of endothelial integrity could also be directly associated with decreased vascular inflammation, since leukocytes may be recruited from the site with endothelial loss by interacting with subendothelial ECMs and smooth muscle cells (48, 49). It should be noted that impairment of endothelial integrity directly exposes the vascular cells to circulating growth factors 

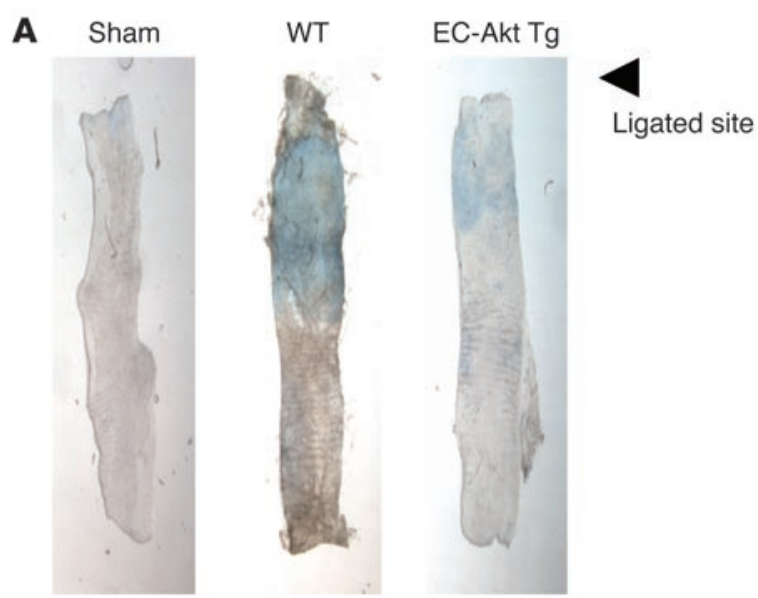

B

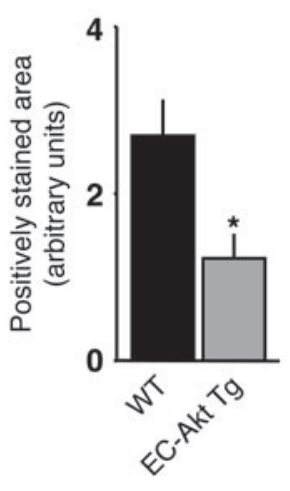

Figure 8

Vascular permeability in EC-Akt Tg mice. (A) Incorporation of Evans blue dye in the vessel wall of sham-operated, WT, and induced EC-Akt Tg mice 1 week after carotid artery ligation. The blue area corresponds to area of increased vascular permeability. (B) Positively stained area of the carotid artery per animal is quantified. Results are expressed as mean \pm SEM. $n=6$ in each group. ${ }^{*} P<0.05$ versus WT.

and cytokines. Taken together, these findings suggest that activation of Akt in the endothelium alone has profound protective effects on the entire vasculature through multiple mechanisms including increased NO production, promotion of EC survival and endothelial integrity, and subsequent suppression of vascular inflammatory and proliferative changes.

Recently, numerous studies have suggested that bone marrow-derived EPCs contribute to neovascularization and vessel repair (50). EPCs participate in re-endothelialization following balloon injury (51). Thus, it is possible that EPCs could be playing a role in our model of vascular injury. However, the possibility of increased Akt activity in EPCs in induced EC-Akt Tg mice appears to be relatively small, since VE-cadherin, an endothelial marker after differentiation, is not expressed abundantly in EPC populations $(50,52)$. Nevertheless, modulating Akt activity in endothelial stem/progenitor cells could be an effective therapeutic strategy for vascular repair, as suggested in previous studies $(35,53)$.

In summary, we have shown that inducible endothelial-specific activation of Akt is sufficient to protect the entire vascular wall after blood flow cessation injury. Since endothelial Akt plays an important role in endothelial function, this pathway may provide a wide spectrum of therapeutic potential in atherosclerotic and ischemic cardiovascular diseases.

\section{Methods}

$\mathrm{Tg}$ mice. All animal experiments were conducted in accordance with approved animal research protocols at Harvard Medical School. For conditional activation of Akt signaling in transgenic mice, we used the tetracycline-controlled gene expression system (tet-off system) where the transgene expression is induced in a tissue-specific manner by 2 transgenes, one for tetracycline-controlled transcriptional activator (tTA) and the other for a gene of interest (16). The tTA gene is driven by the VE-cadherin promoter, which results in endothelial-specific gene expression (54). The transgene for constitutively active, myristoylated Akt 1 (myrAkt) is driven by the promoter provided with a modified tetracycline-responsive element (tet promoter) (17). Double-Tg mice (EC-Akt Tg mice) were obtained by cross-breeding of VE-cadherin-tTA mice with tet-myrAkt mice (15). The construct for the
myrAkt transgene included an HA tag for detection of gene expression. Genotyping of the animals for either transgene was performed as previously described (15). To prevent myrAkt expression in EC-Akt Tg mice, doxycycline $(0.5 \mathrm{mg} / \mathrm{ml} / \mathrm{d}$, orally) in the drinking water was given to pregnant or lactating females, and then to their litters after completion of the lactating period until the appropriate time point for gene induction (doxycycline withdrawal). EC-Akt Tg mice were normal in growth and activity compared with WT or single-Tg mice. For gene induction, doxycycline was withdrawn from the drinking water 3 days before the experiment (15). Some control animals (WT and single-Tg mice) underwent the same protocol for doxycycline administration and withdrawal as the EC-Akt Tg mice did, to exclude possible effects of doxycycline treatment. For all animal experiments, littermates were used as control animals. Systolic blood pressure was measured in the mice by tail-cuff plethysmography at the indicated time points. All of the mice were maintained in the Harvard Medical School animal facilities. The Standing Committee on Animals at Harvard Medical School approved all protocols pertaining to experimentation with the animals.

Cell culture. MLECs were isolated from 4-week-old EC-Akt Tg mice by 2-step immunoselection with PECAM-1- and ICAM-2-conjugated magnetic beads as previously described (55). Cells were cultivated and maintained in DMEM (Invitrogen Corp.) containing 20\% FCS and endothelial growth factors. To suppress myrAkt expression, ECs were maintained in the presence of doxycycline $(500 \mathrm{ng} / \mathrm{ml})$. ECs of passage 2 or 3 were used for all experiments. To avoid heterogeneity of cell population among the groups tested, MLECs isolated from EC-Akt Tg mice were divided into 2 groups, either with or without myrAkt gene induction, and used for comparison. For myrAkt transgene induction, withdrawal of doxycycline from culture medium for 2 days was found to sufficiently induce maximal expression (data not shown).

Measurement of NO production. MLECs were divided into 2 groups, either with or without myrAkt transgene induction, and incubated for 2 days. Nitrite $\left(\mathrm{NO}_{2}\right)$, the stable breakdown product of $\mathrm{NO}$ in aqueous solutions, was measured as previously described (56). After serum starvation for 6 hours, medium was aspirated from culture dishes and replaced with $1 \mathrm{ml}$ fresh serum-free DMEM. After 60 minutes, an aliquot was taken, and the $\mathrm{NO}_{2}$ level was measured using a Sievers $\mathrm{NO}$ analyzer (Analytix Ltd.) and compared with freshly made standards of $\mathrm{NO}_{2}$ in DMEM. Concentrations of $\mathrm{NO}_{2}$ were normalized with the intracellular protein amount in each dish and expressed as nanomoles per milligram.

Organ chamber experiments. The animals were euthanized by i.p. injection of pentobarbital and exsanguinated. Thoracic aorta was carefully dissected and cleaned of any perivascular tissue in cold Krebs solution of the following composition (mM): $\mathrm{NaCl} 121, \mathrm{KCl} 4.7, \mathrm{NaHCO}_{3} 24.7, \mathrm{MgSO}_{4} 12.2$, $\mathrm{CaCl}_{2} 2.5, \mathrm{KH}_{2} \mathrm{PO}_{4} 1.2$, and glucose 5.8. During the procedure, the Krebs solution was aerated with $95 \% \mathrm{O}_{2}$ and $5 \% \mathrm{CO}_{2}$. Isolated aortic rings $(5 \mathrm{~mm})$ were mounted vertically in organ chamber myographs that were filled with Krebs solution. Isometric tension was recorded using a force transducer as previously described $(57,58)$. In order to evaluate the basal release of endothelial NO in EC-Akt Tg mice, contractile response to cumulative concentrations of L-NAME $\left(10^{-7}\right.$ to $\left.10^{-4} \mathrm{M}\right)$ was determined. The extents of contractions were normalized and expressed as percentage of the amplitude of precontractions elicited by saline containing $100 \mathrm{mM} \mathrm{KCl}$.

Western blot analysis. MLECs were divided into 2 groups, either with or without myrAkt transgene induction, and incubated for 2 days. After serum deprivation for 6 hours, MLECs were lysed in cell lysis buffer con- 


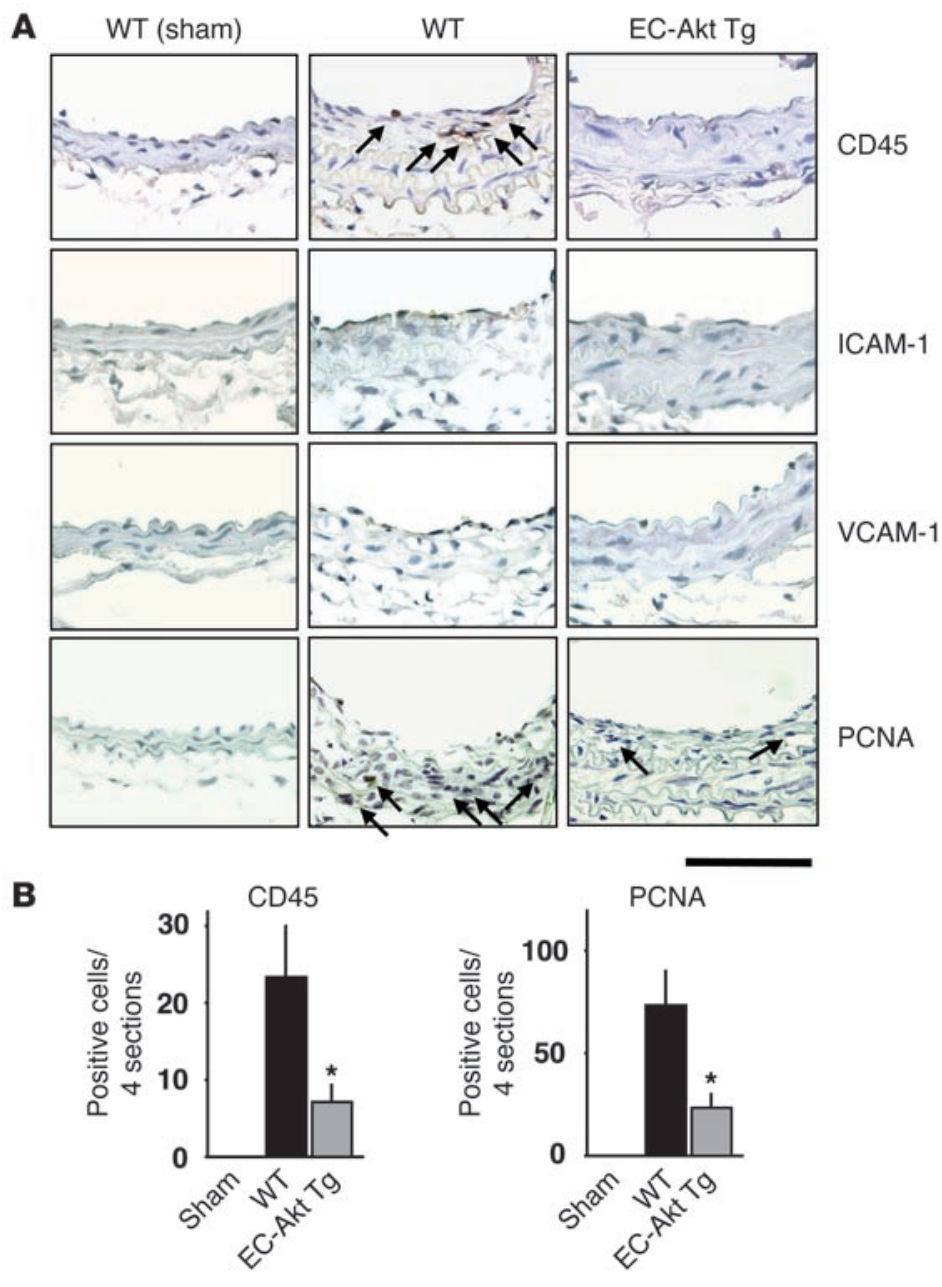

Figure 9

Vascular inflammation following carotid artery ligation. (A) Immunostaining of the vascular wall for leukocyte accumulation (CD45-positive cells), the expression of ICAM-1 and VCAM-1, and cellular proliferation in sham-operated, WT, and induced EC-Akt Tg mice after carotid artery ligation. Arrows indicate positively stained cells. Scale bar: $50 \mu \mathrm{m}$. (B) Quantification of cells that stained positively for CD45 antigen or PCNA. Results are expressed as mean \pm SEM. $n=4$ in each group. ${ }^{*} P<0.05$ versus WT.

taining proteinase inhibitors. In some cells, serum deprivation was done for 24 hours to induce apoptosis and cleaved caspase- 3 expression. An equivalent amount of extracted protein $(10-30 \mu \mathrm{g})$ was loaded for SDSPAGE/immunoblot analysis. HA tag (Amersham Biosciences), total Akt (Cell Signaling Technology), phosphorylated Akt at Ser-473 (Cell Signaling Technology), total GSK-3 $\alpha / \beta$ (Cell Signaling Technology), phosphorylated GSK- $3 \alpha / \beta$ (Cell Signaling Technology), total eNOS (BD Biosciences), phosphorylated eNOS at Ser-1177 (Cell Signaling Technology), and cleaved caspase-3 (Cell Signaling Technology) were detected with each specific antibody. The regions containing the proteins were visualized using an ECL Western blotting luminol reagent (Amersham Biosciences) (56).

Mouse carotid artery ligation model. Male EC-Akt Tg mice ( 6 weeks of age) and their control littermates (WT, single-Tg for tet-myrAkt, or EC-Akt Tg without gene induction) were used for experiments. For EC-Akt Tg mice, expression of myrAkt transgene was induced 3 days before surgery by removal of doxycycline from their drinking water. After anesthesia with ketamine $(80 \mathrm{mg} / \mathrm{kg})$ and xylazine $(5 \mathrm{mg} / \mathrm{kg})$ injected i.p., the left common carotid artery was exposed through a small midline incision in the neck. The artery was completely ligated with a 6-0 silk suture just proximal to the carotid bifurcation to disrupt blood flow (18). In some WT mice, a similar procedure was performed but without ligation (sham operation). In separate groups of animals, L-NAME $(1 \mathrm{mg} / \mathrm{ml})$ was chronically given in the drinking water from 3 days before surgery. The animals were allowed to recover for 4 weeks. All animals recovered completely and showed no neurological deficits.

Morphometric analysis. Four weeks after carotid artery ligation, the animals were euthanized by i.p. injection of ketamine/xylazine. The left ventricle was cannulated and perfused with PBS containing heparin, and then perfused and fixed with $4 \%$ paraformaldehyde in PBS under physiological pressure. Then the left carotid artery was removed. After further incubation in $4 \%$ paraformaldehyde in PBS for 6 hours, the vascular segments were embedded in OCT compound and frozen. Five cryosections ( $6 \mu \mathrm{m}$ thick) at 3-4 mm proximal to the ligation site were obtained in each animal. The 4 different areas (lumen, intima, media, and total vascular area) were measured in sections stained with H\&E using analyzing software (NIH Image 1.62; http://rsb.info.nih.gov/nih-image/download. $\mathrm{html})$ as previously described $(18,19)$. In brief, the areas surrounded by the luminal surface, internal elastic lamina (IEL), and external elastic lamina (EEL) were determined. The intimal area was determined by subtraction of the luminal area from the area defined by the IEL, and the medial area was calculated by subtraction of the area defined by the IEL from the area defined by the EEL. A mean value among 5 sections in each animal was used for analysis.

Immunostaining. One week after ligation, the carotid artery segments were made into frozen cryosections ( $6 \mu \mathrm{m}$ thick) as described above. The sections were stained with the indicated primary antibody overnight at room temperature. The presence of inflammatory cells was determined using an antibody against a common leukocyte antigen, CD45 (1:100 dilution; eBioscience). The expressions of ICAM-1 and VCAM-1 were evaluated using an anti-ICAM-1 antibody (1:50 dilution; Santa Cruz Biotechnology Inc.) and an anti-VCAM-1 antibody (1:100 dilution; Southern Biotechnology Associates Inc.), respectively. PCNA (PCNA staining kit; Zymed Laboratories Inc.) was used to detect proliferating cells in the vascular wall. Subsequently, the sections were incubated with biotinylated secondary antibodies for 60 minutes, and an avidin-biotin-immunoperoxidase system was used for detection. The vascular sections were counterstained with hematoxylin. A mean value of 4 sections per animal was used for analysis.

For detection of cleaved caspase-3, sections were pretreated with $0.1 \%$ Triton X-100 for 10 minutes. An antibody against cleaved (activated) caspase-3 (1:200 dilution; Cell Signaling Technology) was used to detect apoptotic cells $(23,59)$. Then the sections were incubated with a secondary antibody conjugated with FITC (1:200 dilution; Invitrogen Corp.). Thereafter, sections were double-stained with an anti-PECAM-1 antibody (1:200 dilution; BD Biosciences) followed by an Alexa Fluor 546-conjugated secondary antibody (1:200 dilution; Invitrogen Corp.) to visualize the endothelial layer. Using a fluorescence microscope, positively stained ECs were counted. A mean value of 4 sections per animal was used for analysis.

Immunocytochemistry for cleaved caspase-3. MLECs that originated from an EC-Akt Tg mouse were divided into 2 groups, either with or without myrAkt gene induction, and incubated for 2 days. To induce apoptosis, a serum deprivation method for 24 hours was used. Immunofluorescence staining was performed as described previously (60). In brief, cells were fixed and permeabilized with $0.1 \%$ Triton X solution. Expression of cleaved caspase- 3 was determined using a specific antibody (1:200 dilution; Cell Signaling Tech- 
nology) $(23,59)$ followed by a secondary antibody conjugated with FITC (1:200 dilution; Invitrogen Corp.). The HA-tag expression was also determined to confirm myrAkt transgene expression using an antibody (1:100 dilution; Amersham Biosciences) followed by an Alexa Fluor 546-labeled secondary antibody (1:200 dilution; Invitrogen Corp.). Cells were counterstained with Hoechst 33258 for detection of nuclei. Using a fluorescence microscope, cells expressing cleaved caspase- 3 were counted.

In vivo TUNEL assay. One week after ligation, the carotid artery segments were made into frozen cryosections ( $6 \mu \mathrm{m}$ thick) as described above. The sections were incubated in PBS containing $0.1 \%$ Triton X-100 for 10 minutes. Then TUNEL reactions were performed for 60 minutes at $37^{\circ} \mathrm{C}$ using In Situ Cell Death Detection Kit, Fluorescein (Roche Diagnostics Corp.), according to the manufacturer's instructions $(22,40)$. Then sections were double-stained with an anti-PECAM-1 antibody (1:200 dilution; BD Biosciences) followed by an Alexa Fluor 546-conjugated secondary antibody (1:200 dilution) to visualize the EC layer. Using a fluorescence microscope, TUNEL-positive ECs were counted. A mean value of 4 sections per animal was used for analysis.

Vascular permeability assay. Vascular permeability in ligated carotid arteries was studied 1 week after ligation. After heparinization, animals were injected with $50 \mu \mathrm{l}$ solution of $5 \%$ Evans blue dye 30 minutes before euthanasia $(25,61)$. Arteries were perfused with PBS to remove residual blood and the Evans blue dye in the lumen. The arteries were then dissected and opened longitudinally and scanned. Stained area was quantified by NIH Image 1.62 (25).

Statistics. Results were expressed as mean \pm SEM. The data among groups were compared using either 1-way or 2-way ANOVA followed by Bonferroni's post hoc test for multiple comparisons. A $P$ value less than 0.05 was considered to be statistically significant.

\section{Acknowledgments}

This work was supported by grants from the NIH (HL52233, HL70274, DK62729, and HL71049) and the American Heart Association-Bugher Foundation. Y. Mukai is a recipient of a Banyu/ Merck Fellowship Award in Cardiovascular Medicine.

Received for publication July 11, 2005, and accepted in revised form November 29, 2005.

Address correspondence to: James K. Liao, Vascular Medicine Research Unit, Brigham and Women's Hospital, 65 Landsdowne Street, Room 275, Cambridge, Massachusetts 02139, USA. Phone: (617) 768-8424; Fax: (617) 768-8425; E-mail: jliao@rics. bwh.harvard.edu.
1. Shimokawa, H. 1999. Primary endothelial dysfunction: atherosclerosis. J. Mol. Cell. Cardiol. 31:23-37.

2. Landmesser, U., Hornig, B., and Drexler, H. 2004. Endothelial function: a critical determinant in atherosclerosis? Circulation. 109:II27-II33.

3. Bonetti, P.O., Lerman, L.O., and Lerman, A. 2003. Endothelial dysfunction: a marker of atherosclerotic risk. Arterioscler. Thromb. Vasc. Biol. 23:168-175.

4. Melo, L.G., et al. 2004. Endothelium-targeted gene and cell-based therapies for cardiovascular disease. Arterioscler. Thromb. Vasc. Biol. 24:1761-1774.

5. Coffer, P.J., Jin, J., and Woodgett, J.R. 1998. Protein kinase B (c-Akt): a multifunctional mediator of phosphatidylinositol 3-kinase activation. Biochem.J. 335:1-13.

6. Shiojima, I., and Walsh, K. 2002. Role of Akt signaling in vascular homeostasis and angiogenesis. Circ. Res. 90:1243-1250.

7. Fulton, D., et al. 1999. Regulation of endotheliumderived nitric oxide production by the protein kinase Akt. Nature. 399:597-601.

8. Dimmeler, S., et al. 1999. Activation of nitric oxide synthase in endothelial cells by Akt-dependent phosphorylation. Nature. 399:601-605.

9. Rossig, L., Badorff, C., Holzmann, Y., Zeiher, A.M., and Dimmeler, S. 2002. Glycogen synthase kinase-3 couples AKT-dependent signaling to the regulation of p21Cip1 degradation. J. Biol. Chem. 277:9684-9689.

10. Abid, M.R., et al. 2004. Vascular endothelial growth factor activates PI3K/Akt/forkhead signaling in endothelial cells. Arterioscler. Thromb. Vasc. Biol. 24:294-300.

11. Nofer, J.R., et al. 2001. Suppression of endothelial cell apoptosis by high density lipoproteins (HDL) and HDL-associated lysosphingolipids. J. Biol. Chem. 276:34480-34485.

12. Kureishi, Y., et al. 2000. The HMG-CoA reductase inhibitor simvastatin activates the protein kinase Akt and promotes angiogenesis in normocholesterolemic animals. Nat. Med. 6:1004-1010.

13. Kawasaki, K., et al. 2003. Activation of the phosphatidylinositol 3-kinase/protein kinase Akt pathway mediates nitric oxide-induced endothelial cell migration and angiogenesis. Mol. Cell. Biol. 23:5726-5737.

14. Ackah, E., et al. 2005. Akt1/protein kinase $\mathrm{B} \alpha$ is critical for ischemic and VEGF-mediated angio- genesis. J. Clin. Invest. 115:2119-2127. doi:10.1172/ JCI24726.

15. Sun, J.F., et al. 2005. Microvascular patterning is controlled by fine-tuning the Akt signal. Proc. Natl. Acad. Sci. U. S. A. 102:128-133.

16. Mungrue, I.N., et al. 2002. Cardiomyocyte overexpression of iNOS in mice results in peroxynitrite generation, heart block, and sudden death. J. Clin. Invest. 109:735-743.

17. Shiojima, I., et al. 2005. Disruption of coordinated cardiac hypertrophy and angiogenesis contributes to the transition to heart failure. J. Clin. Invest. 115:2108-2118. doi:10.1172/JCI24682.

18. Kumar, A., and Lindner, V. 1997. Remodeling with neointima formation in the mouse carotid artery after cessation of blood flow. Arterioscler. Thromb. Vasc. Biol. 17:2238-2244.

19. Kawashima, S., et al. 2001. Endothelial NO synthase overexpression inhibits lesion formation in mouse model of vascular remodeling. Arterioscler. Thromb. Vasc. Biol. 21:201-207.

20. Dimmeler, S., Assmus, B., Hermann, C., Haendeler, J., and Zeiher, A.M. 1998. Fluid shear stress stimulates phosphorylation of Akt in human endothelial cells: involvement in suppression of apoptosis. Circ. Res. 83:334-341.

21. Jin, Z.G., et al. 2003. Ligand-independent activation of vascular endothelial growth factor receptor 2 by fluid shear stress regulates activation of endothelial nitric oxide synthase. Circ. Res. 93:354-363.

22. Ohashi, H., et al. 2004. Phosphatidylinositol 3-kinase/Akt regulates angiotensin II-induced inhibition of apoptosis in microvascular endothelial cells by governing survivin expression and suppression of caspase-3 activity. Circ. Res. 94:785-793.

23. Limbourg, F.P., et al. 2005. Essential role of endothelial Notch 1 in angiogenesis. Circulation. 111:1826-1832.

24. Nakashima, Y., Raines, E.W., Plump, A.S., Breslow, J.L., and Ross, R. 1998. Upregulation of VCAM-1 and ICAM-1 at atherosclerosis-prone sites on the endothelium in the ApoE-deficient mouse. Arterioscler. Thromb. Vasc. Biol. 18:842-851.

25. Brouchet, L., et al. 2001. Estradiol accelerates reendothelialization in mouse carotid artery through estrogen receptor-alpha but not estrogen receptor-beta. Circulation. 103:423-428.

26. van Beusekom, H.M., et al. 1998. Long-term endothelial dysfunction is more pronounced after stenting than after balloon angioplasty in porcine coronary arteries. J. Am. Coll. Cardiol. 32:1109-1117.

27. Ross, R. 1999. Atherosclerosis is an inflammatory disease [review]. Am. Heart J. 138:S419-S420.

28. Libby, P. 2002. Inflammation in atherosclerosis. Nature. 420:868-874.

29. Wolfrum, S., Jensen, K.S., and Liao, J.K. 2003. Endothelium-dependent effects of statins. Arterioscler. Thromb. Vasc. Biol. 23:729-736.

30. O'Keefe, J.H., Wetzel, M., Moe, R.R., Bronsnahan, K., and Lavie, C.J. 2001. Should an angiotensinconverting enzyme inhibitor be standard therapy for patients with atherosclerotic disease? J. Am. Coll. Cardiol. 37:1-8.

31. Yang, J., et al. 2004. Endothelial overexpression of Fas ligand decreases atherosclerosis in apolipoprotein E-deficient mice. Arterioscler. Thromb. Vasc. Biol. 24:1466-1473.

32. Stabile, E., et al. 2003. Akt controls vascular smooth muscle cell proliferation in vitro and in vivo by delaying G1/S exit. Circ. Res. 93:1059-1065.

33. Asahara, T., et al. 1995. Local delivery of vascular endothelial growth factor accelerates reendothelialization and attenuates intimal hyperplasia in balloon-injured rat carotid artery. Circulation. 91:2793-2801.

34. Hutter, R., et al. 2004. Vascular endothelial growth factor regulates reendothelialization and neointima formation in a mouse model of arterial injury. Circulation. 110:2430-2435.

35. Dimmeler, S., et al. 2001. HMG-CoA reductase inhibitors (statins) increase endothelial progenitor cells via the PI 3-kinase/Akt pathway. J. Clin. Invest. 108:391-397.

36. Zhao, Y.D., Campbell, A.I., Robb, M., Ng, D., and Stewart, D.J. 2003. Protective role of angiopoietin-1 in experimental pulmonary hypertension. Circ. Res. 92:984-991.

37. Nishimatsu, H., et al. 2001. Adrenomedullin induces endothelium-dependent vasorelaxation via the phosphatidylinositol 3-kinase/Akt-dependent pathway in rat aorta. Circ. Res. 89:63-70.

38. Kawai, J., et al. 2004. Endogenous adrenomedullin protects against vascular response to injury in mice. Circulation. 109:1147-1153.

39. Luo, Z., et al. 2000. Acute modulation of endothelial Akt/PKB activity alters nitric oxide- 
dependent vasomotor activity in vivo. J. Clin. Invest. 106:493-499.

40. Tricot, O., et al. 2000. Relation between endothelial cell apoptosis and blood flow direction in human atherosclerotic plaques. Circulation. 101:2450-2453.

41. Rossig, L., Dimmeler, S., and Zeiher, A.M. 2001. Apoptosis in the vascular wall and atherosclerosis. Basic Res. Cardiol. 96:11-22.

42. Shimokawa, H., Flavahan, N.A., Shepherd, J.T., and Vanhoutte, P.M. 1989. Endothelium-dependent inhibition of ergonovine-induced contraction is impaired in porcine coronary arteries with regenerated endothelium. Circulation. 80:643-650.

43. Krejcy, K., et al. 1996. Expression of VCAM-1 in rabbit iliac arteries is associated with vasodilator dysfunction of regenerated endothelium following balloon injury. Atherosclerosis. 122:59-67.

44. Fournet-Bourguignon, M.P., et al. 2000. Phenotypic and functional changes in regenerated porcine coronary endothelial cells: increased uptake of modified LDL and reduced production of NO. Circ. Res. 86:854-861.

45. Dimmeler, S., and Zeiher, A.M. 1999. Nitric oxide: an endothelial cell survival factor. Cell Death Differ. 6:964-968.

46. Kim, W., et al. 2003. Adrenomedullin reduces VEGF-induced endothelial adhesion molecules and adhesiveness through a phosphatidylinositol 3'-kinase pathway. Arterioscler. Thromb. Vasc. Biol. 23:1377-1383.
47. De Caterina, R., et al. 1995. Nitric oxide decreases cytokine-induced endothelial activation. Nitric oxide selectively reduces endothelial expression of adhesion molecules and proinflammatory cytokines. J. Clin. Invest. 96:60-68.

48. Simon, D.I., et al. 2000. Decreased neointimal formation in Mac-1(-/-) mice reveals a role for inflammation in vascular repair after angioplasty. J. Clin. Invest. 105:293-300.

49. Zeiffer, U., et al. 2004. Neointimal smooth muscle cells display a proinflammatory phenotype resulting in increased leukocyte recruitment mediated by P-selectin and chemokines. Circ. Res. 94:776-784.

50. Hristov, M., Erl, W., and Weber, P.C. 2003. Endothelial progenitor cells: mobilization, differentiation, and homing. Arterioscler. Thromb. Vasc. Biol. 23:1185-1189.

51. Griese, D.P., et al. 2003. Isolation and transplantation of autologous circulating endothelial cells into denuded vessels and prosthetic grafts: implications for cell-based vascular therapy. Circulation. 108:2710-2715.

52. Rehman, J., Li, J., Orschell, C.M., and March, K.L. 2003. Peripheral blood "endothelial progenitor cells" are derived from monocyte/macrophages and secrete angiogenic growth factors. Circulation. 107:1164-1169.

53. Mangi, A.A., et al. 2003. Mesenchymal stem cells modified with Akt prevent remodeling and restore performance of infarcted hearts. Nat. Med. 9:1195-1201.
54. Gory, S., et al. 1999. The vascular endothelial-cadherin promoter directs endothelial-specific expression in transgenic mice. Blood. 93:184-192.

55. Lim, Y.C., et al. 2003. Heterogeneity of endothelial cells from different organ sites in T-cell subset recruitment. Am. J. Pathol. 162:1591-1601.

56. Wolfrum, S., et al. 2004. Inhibition of Rho-kinase leads to rapid activation of phosphatidylinositol 3-kinase/protein kinase Akt and cardiovascular protection. Arterioscler. Thromb. Vasc. Biol. 24:1842-1847.

57. Mukai, Y., et al. 2001. Involvement of Rho-kinase in hypertensive vascular disease: a novel therapeutic target in hypertension. FASEB J. 15:1062-1064.

58. Mukai, Y., et al. 2002. Inhibition of renin-angiotensin system ameliorates endothelial dysfunction associated with aging in rats. Arterioscler. Thromb. Vasc. Biol. 22:1445-1450.

59. Gown, A.M., and Willingham, M.C. 2002. Improved detection of apoptotic cells in archival paraffin sections: immunohistochemistry using antibodies to cleaved caspase 3. J. Histochem. Cytochem. 50:449-454.

60. Takemoto, M., et al. 2001. Statins as antioxidant therapy for preventing cardiac myocyte hypertrophy. J. Clin. Invest. 108:1429-1437. doi:10.1172/ JCI200113350.

61. Carmeliet, P., Moons, L., Ploplis, V., Plow, E., and Collen, D. 1997. Impaired arterial neointima formation in mice with disruption of the plasminogen gene. J. Clin. Invest. 99:200-208. 